\section{Original Article}

\section{Corresponding Author}

Alejandro A. Espinoza Orias

(i) https://orcid.org/0000-0002-3792-515X

\author{
Department of Orthopedic Surgery, Rush \\ University Medical Center, 1611 W \\ Harrison Street, Orthopedic Building - \\ Suite 201, Chicago, IL 60612, USA \\ E-mail: Alejandro_Espinoza@rush.edu
}

Received: December 13, 2019

Revised: February 27, 2020

Accepted: February 28, 2020

\section{(c) (i) (2)}

This is an Open Access article distributed under the terms of the Creative Commons Attribution Non-Commercial License (https://creativecommons.org/licenses/by-nc/4.0/) which permits unrestricted non-commercial use, distribution, and reproduction in any medium, provided the original work is properly cited.

Copyright $\odot 2020$ by the Korean Spinal Neurosurgery Society

\title{
Image-Based Markers Predict Dynamic Instability in Lumbar Degenerative Spondylolisthesis
}

\author{
William Slikker III ${ }^{1,2,3}$, Alejandro A. Espinoza Orías ${ }^{1,2}$, Grant D. Shifflett ${ }^{1,2,4}$, \\ Joe Y.B. Lee ${ }^{1,2,5}{\text { Krzysztof Siemionow }{ }^{1,2,6} \text {, Sapan Gandhi }{ }^{1,2} \text {, Louis Fogg }}^{7}$, \\ Dino Samartzis ${ }^{1,2}$, Nozomu Inoue ${ }^{1,2}$, Howard S. An ${ }^{1,2}$ \\ ${ }^{1}$ Department of Orthopedic Surgery, Rush University Medical Center, Chicago, IL, USA \\ ${ }^{2}$ International Spine Research and Innovation Initiative, Rush University Medical Center, Chicago, IL, USA \\ ${ }^{3}$ Private Practice, San Leandro, CA, USA \\ ${ }^{4}$ DISC Sports \& Spine Center, Newport Beach, CA, USA \\ ${ }^{5}$ Private Practice, Arcadia, CA, USA \\ ${ }^{6}$ Department of Orthopedic Surgery, University of Illinois at Chicago, Chicago, IL, USA \\ ${ }^{7}$ College of Nursing, Rush University Medical Center, Chicago, IL, USA
}

Objective: To identify possible radiographic predictors markers of dynamic instability including disc height $(\mathrm{DH})$, disc degeneration, and spondylosis in the setting of degenerative spondylolisthesis (DS).

Methods: A retrospective review with prospectively collected data was performed on 125 patients with L4-5 DS who underwent decompression and fusion. Patients were divided into groups with dynamic instability and those without. Radiographs of the lumbar spine in neutral, flexion, and extension were used to determine degree of slip, $\mathrm{DH}$, translational motion, angular motion, spondylotic changes, and lumbar lordosis. Magnetic resonance imaging (MRI) scans were reviewed to assess disc degeneration.

Results: Thirty-one percent of the patients met criteria for dynamic instability. Significant correlations $(\mathrm{p}<0.05)$ were found between preserved DH and dynamic instability; increased spondylotic changes and decreased translational motion; as well as advanced MRI-based disc degeneration scores with decreased angular motion, respectively. Six radiographic parameters were utilized to create a predictive model for dynamic instability, and a receiver operating characteristic curve was able to validate the predictive model (area $=0.891$, standard error $=0.034, \mathrm{p}<0.001)$.

Conclusion: In DS patients, preserved DH was significantly related to dynamic instability. This finding may represent a greater potential for slip progression over time in these patients. In contrast, disc degeneration on MRI, and spondylotic changes were inversely related to dynamic instability and may represent restabilization mechanisms that decrease the chance of future slip progression in DS.

Keywords: Degenerative spondylolisthesis, Dynamic instability, Intervertebral disc height, Spondylosis

\section{INTRODUCTION}

Although degenerative spondylolisthesis remains one of the most common lumbar pathologies encountered in a clinical spine practice, a complete understanding of its natural history and treatment remains controversial. ${ }^{1}$ Natural history studies following degenerative spondylolisthesis have shown that a subsect of untreated patients experience clinical deterioration, even without radiographic slip progression. ${ }^{2}$ However, the relationship between clinical deterioration, as well as radiographic parameters remain unknown.

Strong evidence supports the role of surgical intervention in 
the setting of degenerative spondylolisthesis patients, with longterm studies showing favorable results over nonsurgical management. ${ }^{3}$ However, the optimal surgical solution remains controversial, particularly regarding the decision of whether or not to perform lumbar arthrodesis in the setting of degenerative spondylolisthesis. ${ }^{1}$ Authors have found differing clinical outcomes regarding the role of lumbar fusion in the setting of degenerative spondylolisthesis, with some finding favorable results with arthrodesis and others finding no advantage compared to decompression alone. ${ }^{4-6}$ From these differing results, it is clear that a more complete understanding of degenerative spondylolisthesis is required to assess its natural progression.

Dynamic instability is the finding of abnormal motion at a spinal level, defined in the literature as greater than $3 \mathrm{~mm}$ of translation or greater than $10^{\circ}$ of motion between adjacent vertebral endplates. ${ }^{7-9}$ From a clinical standpoint, patients with these findings in the setting of degenerative spondylolisthesis may expect to have chronic symptoms of low back and/or leg pain (i.e., greater than 4 years). ${ }^{9}$ Additionally, when deciding on treatment, patients with dynamic instability in the setting of degenerative spondylolisthesis who undergo decompression alone may be at risk of developing postoperative radiographic instability. ${ }^{10}$ These patients may benefit additionally from lumbar fusion, rather than decompression alone. Although the role of dynamic instability in the pathogenesis of degenerative spondylolisthesis remains unclear, identifying patients who have dynamic instability, or may be at risk of developing it, may be vital in selecting the optimal treatment for the patient.

Kirkaldy-Willis and Farfan ${ }^{11}$ suggested that the pathomechanics of lumbar spine degeneration occurs in 3 progressive phases. In the early dysfunction stage, biochemical changes occur and microscopic damage accumulates in the intervertebral disc, along with synovitis of the facet cartilage, which likely occurs prior to the development of spondylolisthesis and/or dynamic instability. As degeneration progresses, there is decreased intervertebral disc height and subluxation of the facet joints leading to increased instability between adjacent vertebral bodies. In the final stage, spondylosis occurs from formation of apophyseal disc osteophytes and the facet joints undergo hypertrophic arthropathy. Their model suggests that dynamic instability and subsequent restabilization of the degenerative lumbar segment occur in separate phases and may linked intimately to disc height as well as disc degeneration. Several studies have linked both disc height and degree of disc degeneration to degree of spondylolisthesis. ${ }^{12,13}$

Given the potential significance of dynamic instability in the pathogenesis of degenerative spondylolisthesis, as well as its potential implications in decision-making in surgical options, the objective of this study was to determine radiographic "predictors" of dynamic instability. We hypothesize that larger disc heights will be correlated with dynamic instability in patients with degenerative spondylolisthesis, while restabilization changes in the form of spondylosis will lead to decreased instability.

\section{MATERIALS AND METHODS}

\section{Study Population}

At our institution, we performed an Institutional Review Boardapproved retrospective review (Study No. ORA 12042303-IRB01) on data from a consecutive series of 125 patients with a known L4-5 degenerative spondylolisthesis diagnosis who underwent decompression and fusion performed by the same surgeon ( $\mathrm{Ta}-$ ble 1). We analyzed standing radiographs of the lumbar spine in neutral, flexion, and extension views as well as magnetic resonance imaging (MRI).

\section{Plain Film Radiography}

On plain radiographs, we assessed the degree of slip distance $(\mathrm{mm})$, disc height at both anterior and posterior edges of the vertebrae $(\mathrm{mm})$, translational motion $(\mathrm{mm})$, angular motion $\left({ }^{\circ}\right)$, and lumbar lordosis (Cobb angle, $\left.{ }^{\circ}\right)$. These variables were measured using electronic templates using a standard medical

Table 1. Patient demographics

\begin{tabular}{lc}
\hline Variable & Value \\
\hline Age $(\mathrm{yr})$ & $69.8 \pm 10.4$ \\
Female sex $(\%)$ & 65 \\
Occupation & Mostly desk work and/or retired \\
Smokers $(\%)$ & 16 \\
L4-5 Cobb angle neutral $\left({ }^{\circ}\right)$ & $19.0 \pm 6.7$ \\
L4-5 Cobb angle extension $\left(^{\circ}\right)$ & $20.4 \pm 7.5$ \\
L4-5 Cobb angle flexion $\left(^{\circ}\right)$ & $16.0 \pm 7.2$ \\
Anterolisthesis in neutral $(\mathrm{mm})$ & $6.7 \pm 3.6$ \\
Anterolisthesis in extension $(\mathrm{mm})$ & $6.3 \pm 4.1$ \\
Anterolisthesis in flexion $(\mathrm{mm})$ & $7.9 \pm 3.8$
\end{tabular}

Values are presented as mean \pm standard deviation unless otherwise indicated.

Statistical differences were found in both radiographic parameters by position: (1) Cobb angle: neutral vs. extension, $\mathrm{p}=0.0003$; neutral vs. flexion, $\mathrm{p}<0.0001$; flexion vs. extension, $\mathrm{p}<0.0001$. (2) Anterolisthesis: neutral vs. extension, $\mathrm{p}=0.0462$; neutral vs. flexion, $\mathrm{p}<0.0001$; flexion vs. extension, $\mathrm{p}<0.0001$. 


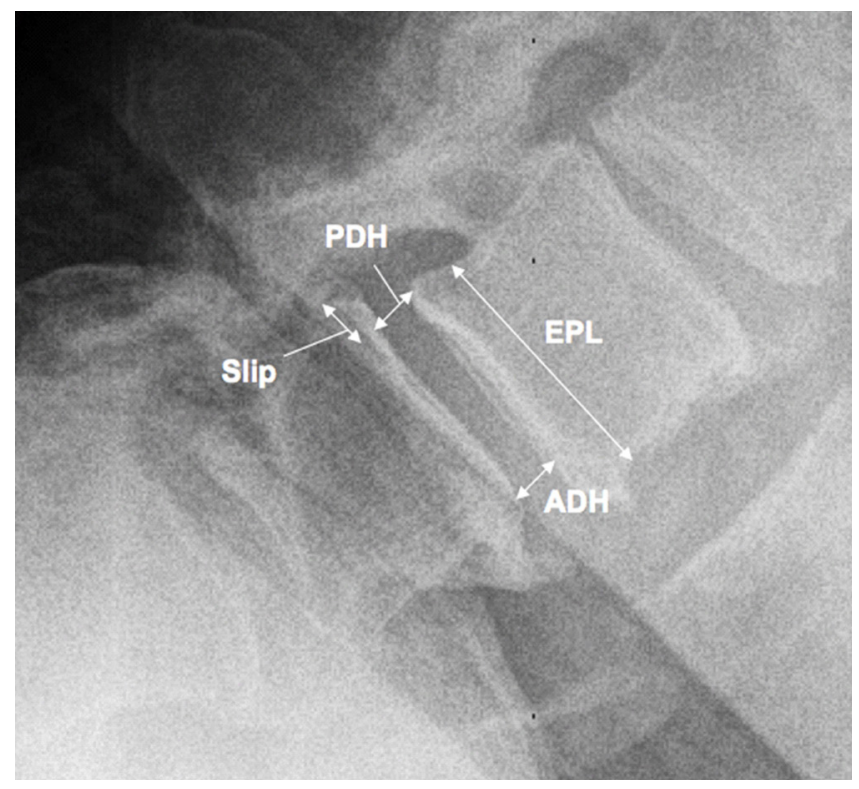

Fig. 1. Measurement of spondylolisthesis. Measurement of Cobb angle, endplate length (EPL), posterior disc height (PDH), anterior disc height (ADH), and slip distance (Slip) were done using plain radiographs in neutral, flexion, and extension using a standard medical image visualization program (Medview, MedImage, Ann Arbor, MI, USA).

image visualization program (Medview, MedImage, Ann Arbor, MI, USA) as shown in Fig. 1. Two individuals, blinded to the study hypothesis, independently assessed all plain radiographs to obtain imaging measurements. Inter- and intrarater reliability analyses were performed. Values by both raters were averaged to obtain a final measurement value. In the event a measurement discrepancy was noted between the raters, a consensus was reached.

\section{Magnetic Resonance Imaging}

MRI was utilized to assess for spondylotic changes and intervertebral disc degeneration. Evidence of spondylosis was determined by the presence of osteophyte spur formation, subcartilaginous sclerosis, and facet hypertrophy at L4-5. In addition, patients who had undergone an MRI as part of their preoperative work-up and had images available for review at the time of this study, were analyzed using the Pfirrmann classification to determine their grade of intervertebral disc degeneration (ranging from grades I to V) at L4-5. ${ }^{14}$ Pfirrman grade 1 refers to homogenous, bright white nucleus, with clear distinction of the nucleus and annulus, and hyper or isointense signal in the nucleus to cerebrospinal fluid. Grade 2 adds inhomogeneity to the nucleus, with or without horizontal bands. Grade 3 refers to a

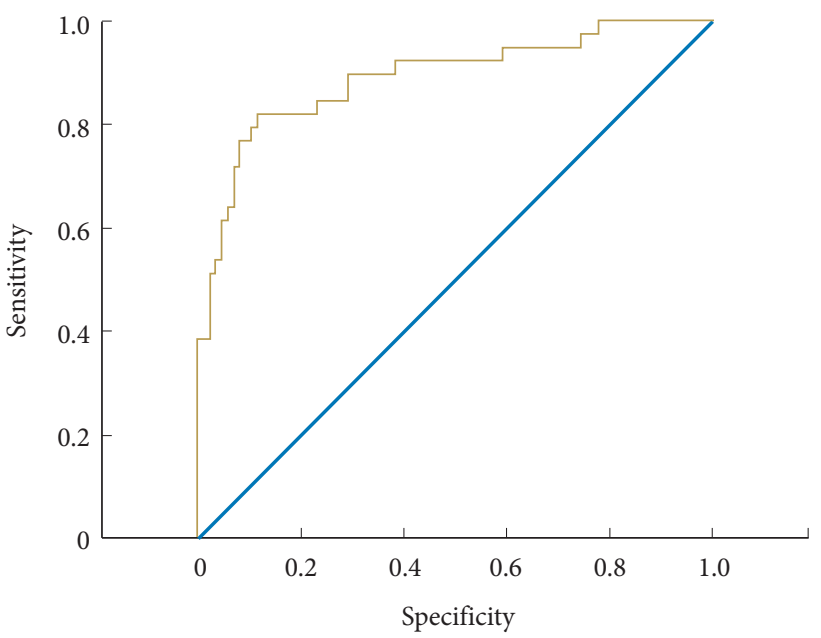

Fig. 2. Receiver operating characteristic (ROC) curve for the spondylolisthesis predictor model presented in this study.

nucleus that is grey and inhomogenous, without clear distinction between the nucleus and annulus, has intermediate signal intensity, and may have slightly decreased disc height. Grade 4 has inhomogenous gray to black nucleus, lost distinction between nucleus and annulus, and moderately decreased disc height. Finally, grade 5 has an inhomogenous black disc, with collapsed disc height. We attempted to evaluate the extent of spondylolisthesis on MRI to compare the degree of disc degeneration with the degree of spondylolisthesis.

\section{Group Stratification}

All patients who were included had degenerative spondylolisthesis, and were further subcategorized into those with dynamic instability or those without dynamic instability. Patients were categorized as having dynamic instability if they had translation of one vertebral body on another in the sagittal plane greater than $3 \mathrm{~mm}$ or greater than $10^{\circ}$ of angular motion between adjacent endplates when comparing flexion and extension radiographs. ${ }^{79}$ Patients with isthmic spondylolisthesis, degenerative spondylolisthesis at other levels, retrolisthesis, lateral listhesis, multilevel spondylolisthesis, or insufficient radiographs were excluded.

\section{Statistical Analyses}

All data was collected, anonymized and recorded on a spreadsheet. IBM SPSS Statistics ver. 22.0 (IBM Co., Armonk, NY, USA) was utilized for the data analyses. Descriptive and frequency statistics were performed of all parameters. Cronbach alpha was utilized to assess inter- and intrarater reliability. An alpha value $\geq 0.90$ was regarded as excellent, whereas values $\geq 0.80$, 
$\geq 70,<70$ were regarded as good, fair and poor, respectively. We used analysis of variance to assess the associations between dynamic instability and radiographic, MRI, and demographic factors. To obtain the statistical model that describes the predictive utility of the variables, a logistic regression was performed using the radiographic parameters measured for all patients to predict unstable spondylolisthesis as opposed to stable spondylolisthesis. A stepwise logistic regression analysis was conducted to determine the optimal set of disc characteristics for predicting unstable spondylolisthesis. A receiver operating characteristic (ROC) curve was used to calculate a relatively optimal cutoff score based on graphical inspection for the optimal predictor index made up of the 6 predictors of stability (Fig. 2). Standard scores associated with the 6 characteristics were summed to create this optimal predictor index (an overall unit-weighted predictor). The optimal predictor index was then used to calculate an area under the curve for the ROC curve. The higher this predictor index is, the more likely the spine is to be unstable. The threshold for statistical significance was established at a pvalue $\leq 0.05$. Odds ratios (ORs) and $95 \%$ confidence intervals were used to assess the strength and precision of the covariates in relation to stability/instability in the logistic regression analyses.

\section{RESULTS}

The study was comprised of 125 patients with degenerative spondylolisthesis at L4-5. Demographics are summarized in Table 1 . The mean age of the subject population was 70 years old, with $65 \%$ being female. The most common occupation was a desk job (36\%) and most patients reported that they were retired at the time of surgery. Only 10 patients in the study had jobs that involved active manual labor at the time of surgery.

Overall, 39 patients (31\%) were found to meet criteria for dynamic instability. The mean amount of anterolisthesis was 6.7 $\mathrm{mm}$ on neutral radiographs, $6.3 \mathrm{~mm}$ on extension radiographs, and $7.9 \mathrm{~mm}$ on flexion radiographs. The mean L4-5 Cobb angle was $19.0^{\circ}$ on neutral radiographs, $20.4^{\circ}$ on extension radiographs and $16.0^{\circ}$ on flexion radiographs. The intraclass analysis noted excellent inter- $(\alpha=0.93)$ and intrarater reliabilities $(\alpha=0.91)$ in relation to slip, disc height, translational motion, angular motion, and lumbar lordosis. Eighty-three of the patients had preoperative MRIs available for review and these were graded using the Pfirrmann grading system. None of the patients had intervertebral discs that could be described as a grade I (i.e., bright signal intensity, nondegenerated disc). From the rest, 7\% had grade II, 35\% had grade III, 29\% had grade IV, and 29\% had grade $\mathrm{V}$ discs, respectively.

We found a significant correlation between preserved disc height and patients with dynamic instability $(\mathrm{p}<0.05)$ (Table 2$)$. Furthermore, there was a significant correlation between advancing disc degeneration on MRI with decreased angular motion $(\mathrm{p}<0.05)$ (Fig. 3). There was no correlation $(\mathrm{r}=-0.12)$ between slip percentage on neutral radiographs and dynamic in-

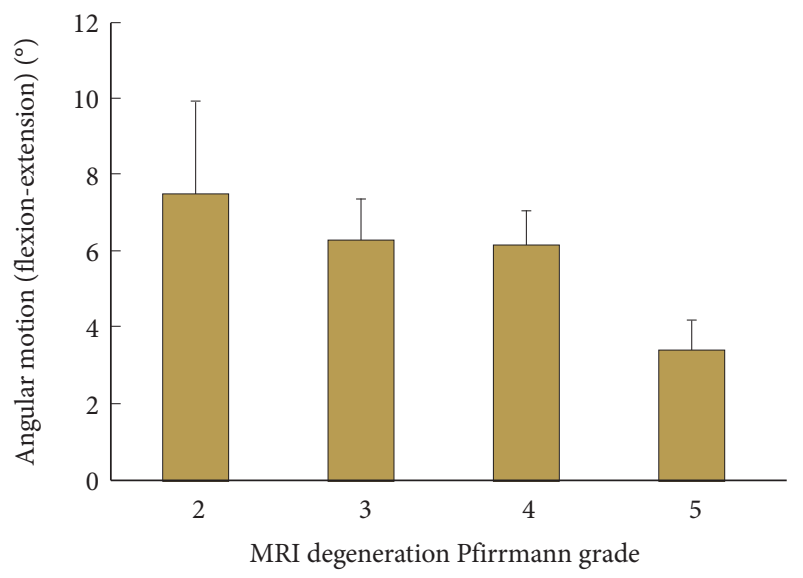

Fig. 3. Angular motion is shown to decrease with advancing magnetic resonance imaging (MRI) disc degeneration Pfirrmann grade. Data are shown as mean \pm standard error of the mean. Significant differences were found between grades 3-5 and $4-5$, respectively.

Table 2. Comparison of patients with dynamic instability to those without: average delta Cobb, translation, and disc height

\begin{tabular}{lcccc}
\hline Variable & $\begin{array}{c}\text { All patients } \\
(\mathrm{n}=125)\end{array}$ & $\begin{array}{c}\text { No dynamic } \\
\text { instability }(\mathrm{n}=86)\end{array}$ & $\begin{array}{c}\text { Dynamic instability }>3 \text { mm of transla- } \\
\text { tion or }>10^{\circ} \text { angular motion }(\mathrm{n}=39)\end{array}$ & $\begin{array}{c}\text { No dynamic instability vs. } \\
\text { instability }(\mathrm{p} \text {-value })\end{array}$ \\
\hline Angular motion $^{*}\left({ }^{\circ}\right)$ & $5.36 \pm 0.42$ & $3.38 \pm 0.26$ & $9.68 \pm 0.88$ & $<0.0001$ \\
Translation $^{\dagger}(\mathrm{mm})$ & $1.92 \pm 0.12$ & $1.39 \pm 0.09$ & $3.07 \pm 0.22$ & $<0.0001$ \\
Anterior disc height $(\mathrm{mm})$ & $10.2 \pm 0.40$ & $9.80 \pm 0.49$ & $11.26 \pm 0.63$ & 0.0862 \\
Posterior disc height $(\mathrm{mm})$ & $5.80 \pm 0.24$ & $5.44 \pm 0.29$ & $6.59 \pm 0.40$ & 0.0245 \\
\hline
\end{tabular}

Values are presented as mean \pm standard error of the mean.

${ }^{*}$ Change in Cobb angle in flexion/extension. ${ }^{\dagger}$ Change in listhesis in flexion/extension. 
Table 3. Variables in the receiver operator curve equation

\begin{tabular}{lcccccc}
\hline Variable & Coefficient & SE & Wald statistic & Significance & Odds ratio & $95 \%$ CI \\
\hline Anterior disc height in extension & 0.596 & 0.187 & 10.142 & 0.001 & 1.815 & $1.258-2.621$ \\
Anterior disc height in flexion & -0.667 & 0.212 & 9.9 & 0.002 & 0.513 & $0.339-0.778$ \\
Posterior disc height in extension & -0.47 & 0.254 & 3.416 & 0.065 & 0.625 & $0.380-1.029$ \\
Posterior disc height in flexion & 0.622 & 0.233 & 7.117 & 0.008 & 1.863 & $1.180-2.944$ \\
Slip in extension & -0.883 & 0.224 & 15.485 & 0 & 0.413 & $0.266-0.642$ \\
Slip in flexion & 1.013 & 0.239 & 17.963 & 0 & 2.753 & $1.724-4.397$ \\
Constant & -5.415 & 1.603 & 11.415 & 0.001 & 0.004 & -
\end{tabular}

SE, standard error; CI, confidence interval.

stability on flexion/extension radiographs. Additionally, lumbar lordosis had weak correlation with slip percentage $(\mathrm{r}=0.21, \mathrm{p}<$ $0.05)$ and with translational motion $(\mathrm{r}=-0.27, \mathrm{p}<0.05)$.

\section{Statistical Model}

The parameters included in the final model included anterior disc height in both extension and flexion, posterior disc height in extension and flexion, slip in extension, and slip in flexion (Table 3). Five of the 6 had significant Wald statistics in the predictive model and 1 (posterior disc height in extension) had a marginal effect $(p=0.065)$. As a consequence, all 6 measures were included in the final predictive model partially for theoretical reasons (leaving it out would have left the model misspecified because an important source of variance would be excluded) and partially for empirical reasons (the sample size was small, making it more difficult for predictors to have sufficient power to be retained, even when they were valid predictors). If the sample were large enough, one could use simple significance as a rule of retaining predictors. This decision was empirically justified by the ROC curve analysis that was used to validate this predictive model. ROC curve is a parallel approach to predicting group membership.

\section{Validation of the Model}

The predictor scores varied from -2.35 to +3.05 . The ROC curve analysis found that this index was an excellent predictor of stability (area $=0.891$, standard error $=0.034, \mathrm{p}<0.001$ ) (Fig. 2). Using the ROC curve, a cutoff score of 0.90 was used to classify participants as likely to be unstable. Participants with scores above the 0.90 cutoff were unstable $89.5 \%$ of the time, while participants with scores below the 0.90 cutoff were unstable only $20.8 \%$ of the time. The differences between the 2 groups were statistically significant (chi-square $=35.45$, degrees of freedom $=1, p<0.001$ ), as one would expect given the highly significant findings associated with the ROC curve analysis.

\section{DISCUSSION}

The presence of dynamic instability in the setting of degenerative spondylolisthesis may have significant implications not only in its pathogenesis, but also the treatment option selected. As such, we conducted a retrospective review of a cohort of 125 patients with degenerative spondylolisthesis, comparing patients with dynamic instability with those that did not, and found that (1) $31 \%$ of patients with degenerative spondylolisthesis have findings of dynamic instability, (2) patients with preserved disc height were more likely to have findings of dynamic instability, (3) increasing levels of lumbar disc degeneration on MRI was inversely related to dynamic instability, (4) lumbar lordosis and lateral L4 5 cobb angle were not associated with dynamic instability, and (5) a logistic model including size of both the anterior and posterior disc height, as well as slip distance, was able in "predicting" the presence of dynamic instability.

In our study, we showed that a larger disc height was correlated with increased dynamic instability. In silico analyses in nonpathologic settings have shown that in increased disc height is associated with greater range of motion at that segment, suggesting that maintained disc height is vital for motion at that level..$^{15}$ In the pathologic setting, Iguchi et al. ${ }^{13}$ examined the effect of age and disc height on findings of segmental translation on radiography in 447 patients and found that disc height was the most intimate factor related to findings of instability. Although Iguchi et al. ${ }^{8}$ did not study a controlled population of patients exclusively of degenerative spondylolisthesis, their definition of instability ( $>3 \mathrm{~mm}$ of translation and $>10^{\circ}$ of angulation) as well as their findings were similar to our study. Both our findings, as well as those of Iguchi et al., ${ }^{12,13}$ are in agreement that increased disc height likely "allows" pathologic instability, particularly in the setting of degenerative spondylolisthesis.

We also found that levels of disc degeneration were inversely 
related to dynamic instability (Fig. 3). In our study, as the Pfirrmann MRI score increases, the amount of translation decreased. These findings are in agreement with other findings in our study (i.e., as disc degeneration progresses, the disc height decreases, and decreases dynamic instability). However, in an MRI study of 70 patients with degenerative disorders of the lumbar spine, Fujiwara et al. ${ }^{16}$ found that increasing levels of disc degeneration corresponded to increased anterior translatory instability. Although their results are in conflict with the present study, Fujiwara et al. ${ }^{16}$ utilized a heterogenous cohort of all degenerative conditions, rather a controlled group of patients with degenerative spondylolisthesis as in our study. In a cohort of 637 patients, Iguchi et al. ${ }^{12}$ found that slip progression of spondylolisthesis was related to the grade of disc degeneration. They found that at grades 3 and higher, spondylolisthesis can progress quickly, leading to further degenerative changes. Although examining a similar patient population to our study, the results of Iguchi et al., ${ }^{12,13}$ focus largely on progression of spondylolisthesis and its relation to disc degeneration, while our findings revolved specifically around dynamic instability in the setting of disc degeneration. All in all, our findings support the notion that dynamic instability is likely decreased in patients with severe degenerative changes due to restabilization mechanisms, such as spondylosis.

Our study found that lumbar lordosis was not correlated with slip distance or with translational motion. Past studies have failed to show a relationship between increased lumbar lordosis and higher risk degenerative spondylolisthesis or slip progression. ${ }^{17}$ Our present study echoed these findings for dynamic instability. In addition, we had hypothesized that the lateral Cobb angle (i.e., segmental alignment) of L4-5 on neutral radiographs may be associated with dynamic instability, but in our present study, that was not the case.

We acknowledge several limitations to this study. We utilized arbitrary designation of dynamic instability with nonvalidated translational and angular motion measurements, which has not been shown to be associated with further progression of spondylolisthesis or nonfusion surgical procedures. Although many clinicians believe that the presence of dynamic instability indicates a necessity to perform spinal fusion when treating spondylolisthesis surgically, there is little evidence to support this notion, outside of the findings of increased radiographic instability after decompression alone. ${ }^{10}$ Degree of disc degeneration, particularly disc height, was found to be associated with dynamic instability in our study, but other factors may play a role in instability or progression, such as osteoporosis, facet joint anat- omy and osteoarthritis, sagittal alignment, pelvic incidence, as well as clinical factors, which were not evaluated in the present study. Consideration of spinopelvic parameters would have additionally strengthened our studies. Additionally, given that patients often obtained MRI and radiography from outside facilities, we did not have standardized imaging parameters. The use of normalized radiograph measurements, as well as inclusion of MRI measurements into the logistic regression, could have strengthened our analysis. Selection of the L4-5 level, as well as only surgical patients, which cannot be applied to degenerative spondylolisthesis at other levels or other patient populations. Finally, the results of this study cannot be applied to predict clinical or radiographic progression following conservative or operative treatment. Future prospective longitudinal studies are needed to determine the exact contribution of dynamic instability to the progression of degenerative spondylolisthesis, as well as its effect in surgical decision-making.

\section{CONCLUSION}

In patients with degenerative spondylolisthesis, preserved disc height was associated with dynamic instability, while increasing levels of disc degeneration were inversely related to dynamic instability. These findings may provide insight into radiographic parameters that may predict the presence of dynamic instability, as well as suggest that restabilization mechanisms may halt this motion. Future studies should focus on prospective, longitudinal study designs to examine the role of dynamic instability in the pathogenesis and progression of degenerative spondylolisthesis. Additionally, future investigations are warranted to elucidate the role of dynamic instability in outcome following lumbar fusion for degenerative spondylolisthesis.

\section{CONFLICT OF INTEREST}

The authors have nothing to disclose.

\section{REFERENCES}

1. Kleinstueck FS, Fekete TF, Mannion AF, et al. To fuse or not to fuse in lumbar degenerative spondylolisthesis: do baseline symptoms help provide the answer? Eur Spine J 2012; 21:268-75

2. Matsunaga S, Sakou T, Morizono Y, et al. Natural history of degenerative spondylolisthesis. Pathogenesis and natural course of the slippage. Spine (Phila Pa 1976) 1990;15:1204- 
10.

3. Weinstein JN, Lurie JD, Tosteson TD, et al. Surgical compared with nonoperative treatment for lumbar degenerative spondylolisthesis. four-year results in the Spine Patient Outcomes Research Trial (SPORT) randomized and observational cohorts. J Bone Joint Surg Am 2009;91:1295-304.

4. Försth P, Ólafsson G, Carlsson T, et al. A randomized, controlled trial of fusion surgery for lumbar spinal stenosis. $\mathrm{N}$ Engl J Med 2016;374:1413-23.

5. Ghogawala Z, Dziura J, Butler WE, et al. Laminectomy plus fusion versus laminectomy alone for lumbar spondylolisthesis. N Engl J Med 2016;374:1424-34.

6. Herkowitz HN, Kurz LT. Degenerative lumbar spondylolisthesis with spinal stenosis. A prospective study comparing decompression with decompression and intertransverse process arthrodesis. J Bone Joint Surg Am 1991;73:802-8.

7. Boden SD, Wiesel SW. Lumbosacral segmental motion in normal individuals. Have we been measuring instability properly? Spine (Phila Pa 1976) 1990;15:571-6.

8. Iguchi T, Kanemura A, Kasahara K, et al. Age distribution of three radiologic factors for lumbar instability: probable aging process of the instability with disc degeneration. Spine (Phila Pa 1976) 2003;28:2628-33.

9. Kanemura A, Doita M, Kasahara K, et al. The influence of sagittal instability factors on clinical lumbar spinal symptoms. J Spinal Disord Tech 2009;22:479-85.

10. Fox MW, Onofrio BM, Onofrio BM, et al. Clinical outcomes and radiological instability following decompressive lumbar laminectomy for degenerative spinal stenosis: a comparison of patients undergoing concomitant arthrodesis versus decompression alone. J Neurosurg 1996;85:793-802.

11. Kirkaldy-Willis WH, Farfan HF. Instability of the lumbar spine. Clin Orthop Relat Res 1982;(165):110-23.

12. Iguchi $T$, Nishida $K$, Ozaki $T$, et al. Grade three disc degeneration is a critical stage for anterior spondylolisthesis in lumbar spine. Eur Spine J 2012;21:2134-9.

13. Iguchi $\mathrm{T}$, Ozaki $\mathrm{T}$, Chin $\mathrm{T}$, et al. Intimate relationship between instability and degenerative signs at $\mathrm{L} 4 / 5$ segment examined by flexion-extension radiography. Eur Spine J 2011;20:134954.

14. Pfirrmann CW, Metzdorf A, Zanetti M, et al. Magnetic resonance classification of lumbar intervertebral disc degeneration. Spine (Phila Pa 1976) 2001;26:1873-8.

15. Natarajan RN, Andersson GB. The influence of lumbar disc height and cross-sectional area on the mechanical response of the disc to physiologic loading. Spine (Phila Pa 1976) 1999; 24:1873-81.

16. Fujiwara A, Tamai K, An HS, et al. The relationship between disc degeneration, facet joint osteoarthritis, and stability of the degenerative lumbar spine. J Spinal Disord 2000;13:44450 .

17. Berlemann U, Jeszenszky DJ, Bühler DW, et al. The role of lumbar lordosis, vertebral end-plate inclination, disc height, and facet orientation in degenerative spondylolisthesis. J Spinal Disord 1999;12:68-73. 